\title{
Changes in metabolic parameters and adverse kidney and cardiovascular events during glomerulonephritis and renal vasculitis treatment in patients with and without diabetes mellitus
}

\author{
Cynthia C. Lim, Jason C. J. Choo, Hui Zhuan Tan, Irene Y. J. Mok, Yok Mooi Chin, Choong Meng Chan, \\ Keng Thye Woo
}

Department of Renal Medicine, Singapore General Hospital, Singapore

\begin{abstract}
Background: Cardiovascular disease causes significant morbidity and mortality in patients with glomerulonephritis, which is increasingly diagnosed in older individuals who may have diabetes mellitus (DM). We evaluated the impact of DM on metabolic profile, renal and cardiovascular outcomes during treatment and follow-up of individuals with glomerulonephritis.

Methods: We performed a retrospective cohort study of 601 consecutive adults with biopsy-proven glomerulonephritis for factors associated with kidney failure, hospitalization for cardiovascular events, and death. Biopsies with isolated diabetic nephropathy were excluded.

Results: The median patient age was 49.8 years (36.7-60.9 years) with estimated glomerular filtration rate of $56.7 \mathrm{~mL} / \mathrm{min} / 1.73$ $\mathrm{m}^{2}$ (27.7-93.2 mL/min/1.73 $\left.\mathrm{m}^{2}\right)$. DM was present in 25.4\%. The most frequent diagnoses were minimal change disease (MCD) or focal segmental glomerulosclerosis (FSGS) (29.5\%), lupus nephritis (21.3\%), immunoglobulin A (IgA) nephropathy (19.1\%), and membranous nephropathy (12.1\%). The median follow-up was 38.8 months (interquartile range [IQR], 26.8-55.8 months). Among 511 individuals with lupus nephritis, anti-neutrophil cytoplasmic antibody-associated vasculitis, MCD/FSGS, membranous nephropathy, and IgA nephropathy, 52 (10.2\%) developed kidney failure at a median 16.4 months (IQR, 2.3-32.2 months), while 29 (5.7\%) had cardiovascular-related hospitalizations at 12.9 months (IQR, 4.8-31.8 months) and 31 (6.1\%) died at 13.5 months (IQR, $2.5-42.9$ months) after diagnosis. Cox regression analysis found that baseline DM was independently associated with kidney failure (adjusted hazard ratio [HR], 2.07; 95\% confidence interval [Cl], 1.06-4.05, $\mathrm{p}=0.03$ ) and cardiovascular-related hospitalization (adjusted HR, 2.69; $95 \% \mathrm{Cl}, 1.21-5.98, \mathrm{p}=0.02$ ) but not with mortality.

Conclusion: DM was strongly associated with kidney failure and hospitalization for cardiovascular events in patients with biopsy-proven glomerulonephritis.
\end{abstract}

Keywords: Cardiovascular diseases, Diabetes mellitus, Glomerulonephritis, Renal insufficiency

Received: September 18, 2020; Revised: December 21, 2020; Accepted: January 17, 2021

Editor: Soon Hyo Kwon, Soonchunhyang University, Seoul, Republic of Korea

Correspondence: Cynthia C. Lim

Department of Renal Medicine, Singapore General Hospital, Academia Level 3, 20 College Road, Singapore 169856, Singapore. E-mail: cynthia.lim. c.w@singhealth.com.sg

ORCID: https://orcid.org/0000-0003-0021-4861

Copyright (c) 2021 by The Korean Society of Nephrology

(a) This is an Open Access article distributed under the terms of the Creative Commons Attribution Non-Commercial and No Derivatives License (http:// creativecommons.org/licenses/by-nc-nd/4.0/) which permits unrestricted non-commercial use, distribution of the material without any modifications, and reproduction in any medium, provided the original works properly cited. 


\section{Introduction}

Glomerulonephritis and renal vasculitis continue to be frequent causes of end-stage kidney disease (ESKD) worldwide [1-3], and mortality from acute and chronic glomerulonephritis rose $25 \%$ over the past 10 years [4]. Individuals with glomerulonephritis are increasingly older [5-9] and are more likely to have metabolic diseases that are associated with older age, such as diabetes mellitus (DM) and hypertension [7,9]. In addition, treatment with immunosuppressants such as glucocorticosteroids may cause or exacerbate DM, hypertension, and obesity [10-12]. These conditions are established cardiovascular risk factors $[13,14]$, while certain systemic diseases that cause glomerulonephritis, such as anti-neutrophil cytoplasmic antibody (ANCA)-associated vasculitis, may be intrinsically associated with increased cardiovascular risk $[15,16]$. Cardiovascular disease is a major contributor to morbidity and mortality in patients with glomerulonephritis [16-20]. Given the propensity for metabolic and cardiovascular disease among individuals with glomerulonephritis and renal vasculitis, we aimed to evaluate the impact of DM on the metabolic profile, as well as adverse renal and cardiovascular outcomes during treatment and follow-up of individuals with glomerulonephritis.

\section{Methods}

This was a retrospective cohort study of consecutive adults aged $\geq 21$ years with biopsy-proven glomerulonephritis and renal vasculitis diagnosed between January 2011 and July 2015 at the Singapore General Hospital, an academic medical center and tertiary referral center. Subjects were identified from the kidney biopsy procedural log, which recorded all native kidney biopsies performed. Biopsies with isolated diabetic nephropathy $(n=64)$ were excluded. This study was conducted according to the guidelines of the Declaration of Helsinki and was approved by the local Institutional Review Board (No. 2015/2882). The requirement for informed consent was waived because of the retrospective nature of the study.

Demographic, comorbid disease, laboratory data, and medication data were retrieved from electronic medical records. Presence of DM at presentation was defined according to physician diagnosis of DM, fasting glucose $\geq 7$ $\mathrm{mmol} / \mathrm{L}$, glycated hemoglobin (HbAlc) $\geq 6.5 \%$, or when glucose-lowering medications were required. Baseline hyper- tension, hyperlipidemia, and ischemic heart disease were identified from medical records. Laboratory data collected included serum creatinine and urine protein-to-creatinine ratio (UPCR) within 1 month before kidney biopsy; fasting triglyceride (TG), high-density lipoprotein cholesterol and low-density lipoprotein cholesterol (LDL-C) within 24 months preceding biopsy and within 6 months after immunosuppressant; and $\mathrm{HbAlc}$ and fasting glucose within 6 months before and after immunosuppressant. Estimated glomerular filtration rate (eGFR) was calculated using the Chronic Kidney Disease Epidemiology Collaboration (CKDEPI) equation [21]. Nephrotic-range proteinuria was defined if UPCR was greater than $3 \mathrm{~g} / \mathrm{g}$. All laboratory investigations were conducted at our center, the laboratory of which is accredited by the College of American Pathologists. Pharmacotherapy data retrieved included angiotensin converting enzyme (ACE) inhibitor, angiotensin II receptor blocker (ARB), antidiabetic, antilipid, and immunosuppressant medication (glucocorticosteroid, calcineurin inhibitor, mycophenolate mofetil or sodium, cyclophosphamide, and azathioprine) prior to and after kidney biopsy, as well as peak daily dose of each immunosuppressant after diagnosis of biopsy-confirmed glomerulonephritis.

We assessed (1) changes in glycemic and lipid indices and need for antidiabetic therapy during therapy for glomerulonephritis and renal vasculitis; (2) occurrence and time to ESKD, defined by need for dialysis or transplant or if serum creatinine was $>500 \mu \mathrm{mol} / \mathrm{L}$; (3) hospitalization for cardiovascular events including acute myocardial infarct, congestive cardiac failure or cardiac catheterization showing $>50 \%$ coronary artery stenosis; and (4) death. Data were retrieved until last hospital visit or death.

Statistical analyses were performed using IBM SPSS version 26 (IBM Corp., Armonk, NY, USA). Categorical variables were presented as proportions and continuous variables were summarized as medians with interquartile ranges (IQR [25th percentile-75th percentile]). The baseline clinical characteristics, metabolic profiles, and medications were compared for individuals with and without DM using the Pearson chi-square test for categorical variables and the Mann-Whitney U test for non-normally distributed continuous variables. When analyzing factors associated with clinical outcomes, we included the most frequent diagnoses (minimal change disease [MCD] or focal segmental glomerulosclerosis [FSGS], lupus nephritis, immunoglobulin A (IgA) 
nephropathy, membranous nephropathy, and ANCA-associated vasculitis) and excluded patients with "other diagnoses" in order to reduce the heterogeneity of the study cohort. Kaplan-Meier survival curves for the clinical outcomes were compared using log-rank tests. Cox regression (stepwise method) was performed to obtain hazard ratios (HRs) and 95\% confidence intervals (CIs) for factors that were independently associated with the clinical outcomes.

Covariates (age, sex, glomerulonephritis diagnosis, DM, hypertension, hyperlipidemia, ischemic heart disease, eGFR, UPCR, use of ACE inhibitor or ARB, and immunosuppressant treatment after biopsy) were chosen a priori. Multicollinearity was checked by examining the correlation matrix for coefficient values of $\geq 0.80$. Stratified analyses to test for associations between DM and clinical outcomes were performed according to age ( $<50$ years vs. $\geq 50$ years), sex (female vs. male), ischemic heart disease (no vs. yes), eGFR category $\left(\geq 60 \mathrm{~mL} / \mathrm{min} / 1.73 \mathrm{~m}^{2}\right.$ vs. $\left.<60 \mathrm{~mL} / \mathrm{min} / 1.73 \mathrm{~m}^{2}\right)$, UPCR category ( $<3 \mathrm{~g} / \mathrm{g}$ vs. $\geq 3 \mathrm{~g} / \mathrm{g}$ ), use of ACE inhibitor or ARB (no vs. yes), and immunosuppressant use (no vs. yes). The p-values of interactions for effect modification were obtained by including the interaction term ( $\mathrm{DM}^{*}$ variable) in the regression model. Subgroup analyses were performed for those with and without DM. All tests were two-tailed and statistical significance was defined as $\mathrm{p}<0.05$.

\section{Results}

\section{Cohort clinical characteristics}

We evaluated 601 individuals with biopsy-proven glomerulonephritis and renal vasculitis. Our multiethnic Southeast Asian cohort included Chinese (464 patients, $77.2 \%)$, Malay (77, 12.8\%), Indian (22, 3.7\%), and other ethnicities (38, 6.3\%). The median age was 49.8 years (36.7-60.9 years) with eGFR $56.7 \mathrm{~mL} /$ $\mathrm{min} / 1.73 \mathrm{~m}^{2}\left(27.7-93.2 \mathrm{~mL} / \mathrm{min} / 1.73 \mathrm{~m}^{2}\right)$. DM was present in 153 (25.5\%) at biopsy. The most frequent diagnoses were MCD or FSGS (177 patients, 29.5\%), lupus nephritis (128, 21.3\%), IgA nephropathy $(115,19.1 \%)$, and membranous nephropathy (73, $12.1 \%)$. Eighteen patients (3.0\%) had ANCA-associated vasculitis while other diagnoses (90 patients, 15.0\%) included thin membrane disease, Alport disease, ANCA-negative pauci-immune vasculitis, undifferentiated immune-complex mediated glomerulonephritis, antiglomerular basement membrane disease, and tubulointerstitial nephritis.
Histological features of diabetic nephropathy were present concurrently with glomerulonephritis in 34 biopsies (5.7\%); 22 minimal changes or FSGS, three IgA nephropathies, two membranous nephropathies, one each of lupus nephritis and ANCA-associated vasculitis, and five others.

Table 1 compares the clinical characteristics and pharmacotherapy of patients with and without DM at presentation. Those with DM were older, more likely to be male, and to have hypertension, hyperlipidemia, and ischemic heart disease. They also had lower eGFR and greater proteinuria. Individuals with DM were more likely to have received blood pressurelowering medications, including ACE inhibitor and ARB, before biopsy. After diagnosing biopsy-proven glomerulonephritis, treatment with ACE inhibitor or ARB did not differ between groups. Individuals with DM were less likely to receive immunosuppressive therapy such as glucocorticosteroid, but the peak daily dose and cumulative treatment duration of prednisolone and the use and treatment duration of calcineurin inhibitor were not different between groups.

\section{Effect of baseline diabetes mellitus on metabolic profile after immunosuppressive therapy}

Table 2 describes the metabolic profiles of 408 individuals who received immunosuppressant treatment. During the 6-month follow-up after starting immunosuppressant treatment, fasting glucose, TG, and LDL-C results were available for 294 individuals, while $\mathrm{HbAlc}$ was available for 92 individuals. Patients with DM were more likely to have increased TG and LDL-C after immunosuppressant, compared to individuals without DM. Antidiabetic and antilipid medications at baseline and after immunosuppressive therapy were also more frequently prescribed among those with DM. Fig. 1 shows that the need for oral antidiabetic therapy nearly doubled and the need for insulin therapy increased four-fold among individuals with DM treated with immunosuppressants. The need for antidiabetic medications decreased over time in both DM (Fig. 1A) and non-DM (Fig. 1B) patients, possibly due to tapering doses of induction immunosuppressant.

\section{Effect of baseline diabetes mellitus on clinical outcomes}

Among 511 individuals with lupus nephritis, ANCA-associated vasculitis, MCD/FSGS, membranous nephropathy, and IgA nephropathy, 52 (10.2\%) developed ESKD at a median of 16.4 
Table 1. Clinical characteristics and treatment of 601 individuals with biopsy-proven glomerulonephritis and renal vasculitis according to presence of DM at baseline

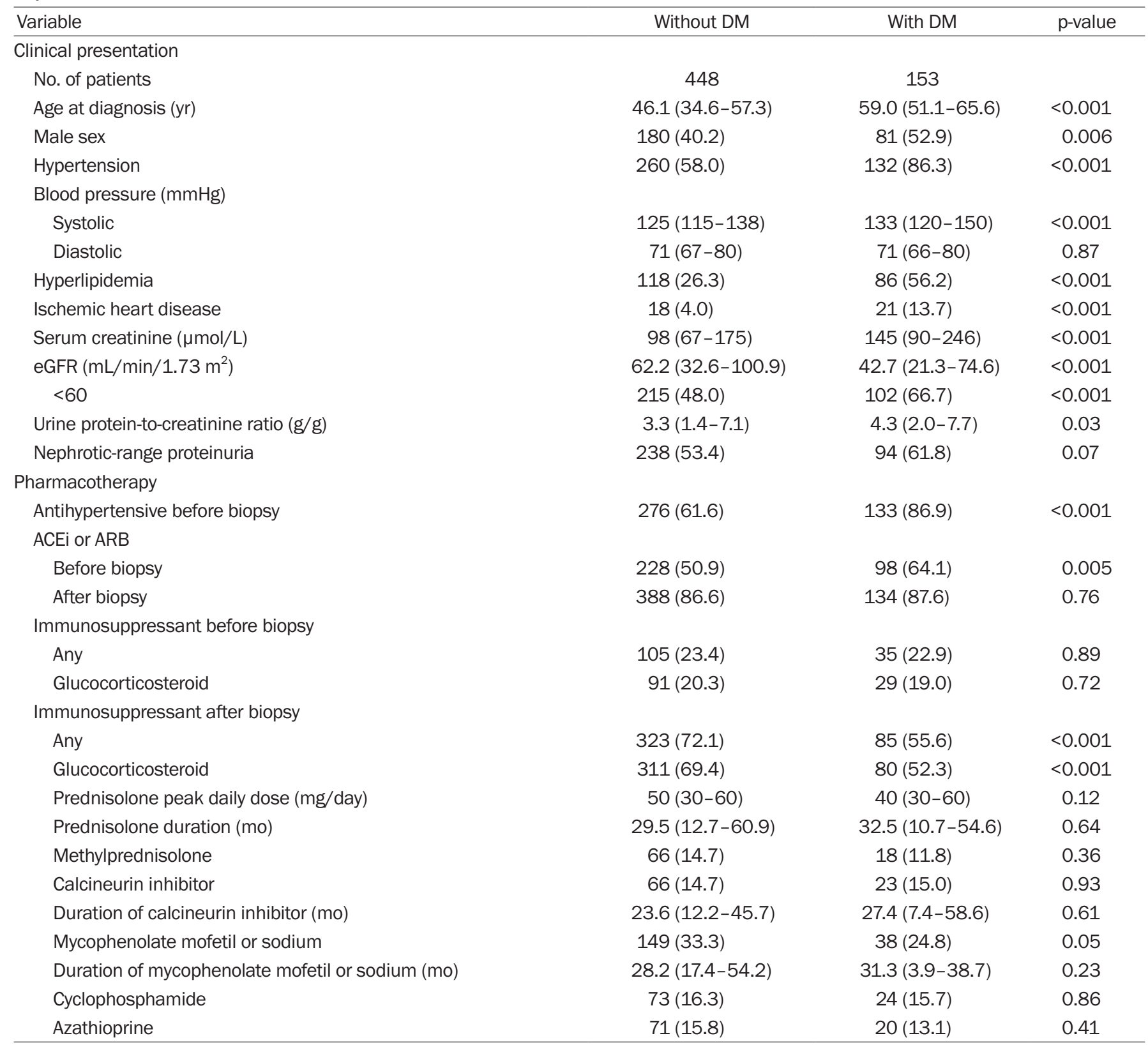

Data are expressed as number only, median (interquartile range), or number (\%).

$\mathrm{ACEi}$, angiotensin converting enzyme inhibitor; ARB, angiotensin II receptor blocker; eGFR, estimated glomerular filtration rate calculated using the Chronic Kidney Disease Epidemiology Collaboration equation; DM, diabetes mellitus.

months (IQR, 2.3-32.2 months), while 29 (5.7\%) had cardiovascular-related hospitalizations at a median of 12.9 months (4.8-31.8 months), and 31 (6.1\%) died at a median 13.5 months (2.5-42.9 months) after diagnosis.

The follow-up duration was comparable between those with and without DM (median of 36.5 months [IQR, 24.453.8 months] vs. 40.0 months [IQR, 27.3-57.2 months], re- spectively; $\mathrm{p}=0.31)$. In the DM group $(\mathrm{n}=120)$, there were 21 ESKD, 18 cardiovascular-related hospitalizations, and 10 deaths. In the no-DM group ( $\mathrm{n}=391)$, there were $31 \mathrm{ESKD}$, 11 cardiovascular-related hospitalizations, and 21 deaths. Individuals with DM at baseline were more likely to develop ESKD ( $17.5 \%$ vs. $7.9 \%, \mathrm{p}=0.002)$ and to experience a cardiovascular event requiring hospitalization $(15.0 \%$ vs. $2.8 \%$, 
Table 2. Metabolic profiles at baseline and after immunosuppressive therapy

\begin{tabular}{|c|c|c|c|}
\hline \multirow{2}{*}{ Variable } & \multicolumn{3}{|c|}{$\begin{array}{l}\text { Biopsy-proven glomerulonephritis and renal vasculitis treated with } \\
\text { immunosuppressants }(n=408)\end{array}$} \\
\hline & Without DM $(n=323)$ & With DM $(n=85)$ & p-value \\
\hline \multicolumn{4}{|l|}{ Antidiabetic medication } \\
\hline At baseline & $0(0)$ & 40 (47.1) & $<0.001$ \\
\hline After immunosuppressant & $19(5.9)$ & $62(72.9)$ & $<0.001$ \\
\hline \multicolumn{4}{|l|}{ Antilipid medication } \\
\hline After immunosuppressant & $213(65.9)$ & $70(83.4)$ & 0.002 \\
\hline \multicolumn{4}{|l|}{ Fasting glucose } \\
\hline At baseline (mmol/L) & $5.1(4.8-5.7)$ & $7.0(5.8-8.2)$ & $<0.001$ \\
\hline Peak value after immunosuppressant ${ }^{a}$ (mmol/L) & $5.6(4.9-6.8)$ & $7.8(6.6-9.5)$ & $<0.001$ \\
\hline Change from baseline (\%) & $9.6(-4.2$ to 33.2$)$ & $10.0(-15.2$ to 45.5$)$ & 0.76 \\
\hline Change from baseline (\%) & $2.6(0-13.6)$ & $8.0(-3.1$ to 33.6$)$ & 0.40 \\
\hline \multicolumn{4}{|l|}{ Fasting triglyceride } \\
\hline At baseline (mmol/L) & $1.69(1.20-2.30)$ & $1.73(1.19-2.50)$ & 0.79 \\
\hline Peak value after immunosuppressant ${ }^{a}$ (mmol/L) & $1.43(1.03-2.05)$ & $1.93(1.22-2.45)$ & 0.004 \\
\hline Change from baseline (\%) & $-11.2(-42.9$ to 21.4$)$ & $5.0(-23.9$ to 42.3$)$ & 0.01 \\
\hline \multicolumn{4}{|l|}{ Fasting LDL-cholesterol } \\
\hline At baseline (mmol/L) & $3.93(2.91-6.10)$ & $3.10(2.24-4.21)$ & 0.001 \\
\hline Peak value after immunosuppressant ${ }^{a}(\mathrm{mmol} / \mathrm{L})$ & $3.36(2.67-4.16)$ & $3.21(2.27-4.23)$ & 0.29 \\
\hline Change from baseline (\%) & $-14.3(-52.2$ to 15.4$)$ & $4.0(-35.6$ to 51.4$)$ & 0.006 \\
\hline
\end{tabular}

Data are expressed as number (\%) or median (interquartile range).

DM, diabetes mellitus; HbA1c, glycated hemoglobin; LDL, low-density lipoprotein

${ }^{a}$ Fasting glucose, triglyceride, and LDL-cholesterol results were available for 294 individuals during the initial 6 months after immunosuppressants were started. ${ }^{\mathrm{b}} \mathrm{HbA} 1 \mathrm{c}$ was available for 92 individuals during the initial 6 months after immunosuppressants were started.

$\mathrm{p}<0.001)$. Mortality was not significantly different between the groups ( $8.3 \%$ vs. $5.4 \%, \mathrm{p}=0.24$ ). Survival analyses (Fig. 2) showed that the cumulative probabilities of renal survival and survival without cardiovascular-related hospitalization were significantly lower in those with DM at baseline. Multivariate analyses shown in Tables 3 and 4 confirmed that DM was independently associated with ESKD (adjusted HR, 2.07; 95\% CI, 1.06-4.05; $\mathrm{p}=0.03$ ) and cardiovascular-related hospitalization (adjusted HR, 2.69; 95\% CI, 1.21-5.98; p $=0.02$ ) but not with mortality. Interactions were observed between DM and proteinuria for ESKD, and for DM and sex for cardiovascular-related hospitalizations (Supplementary Table 1, available online): DM was associated with increased ESKD in patients with nephrotic-range proteinuria (HR, 2.33; 95\% CI, 1.18-4.63) but not in those without nephrotic-range proteinuria (HR, 2.86; 95\% CI, 0.86-9.54); DM was associated with increased cardiovascular-related hospitalization in both female (HR, 6.92; 95\% CI, 1.95-24.57) and male patients (HR, 3.97; 95\% CI, 1.54-10.26), but by varying degrees.

\section{Other factors associated with end-stage kidney disease, cardiovascular-related hospitalization, and mortality}

Multivariate analyses for ESKD, cardiovascular-related hospitalization, and death are shown in Tables 3 and 4. Older age was independently associated with increased risk of mortality but reduced risk of ESKD. Male sex increased the risks of cardiovascular-related hospitalization and death. Compared to IgA nephropathy, both lupus nephritis and ANCA-associated vasculitis independently increased the risks of cardiovascular-related hospitalization and death, but while patients with ANCA-associated vasculitis had greater 

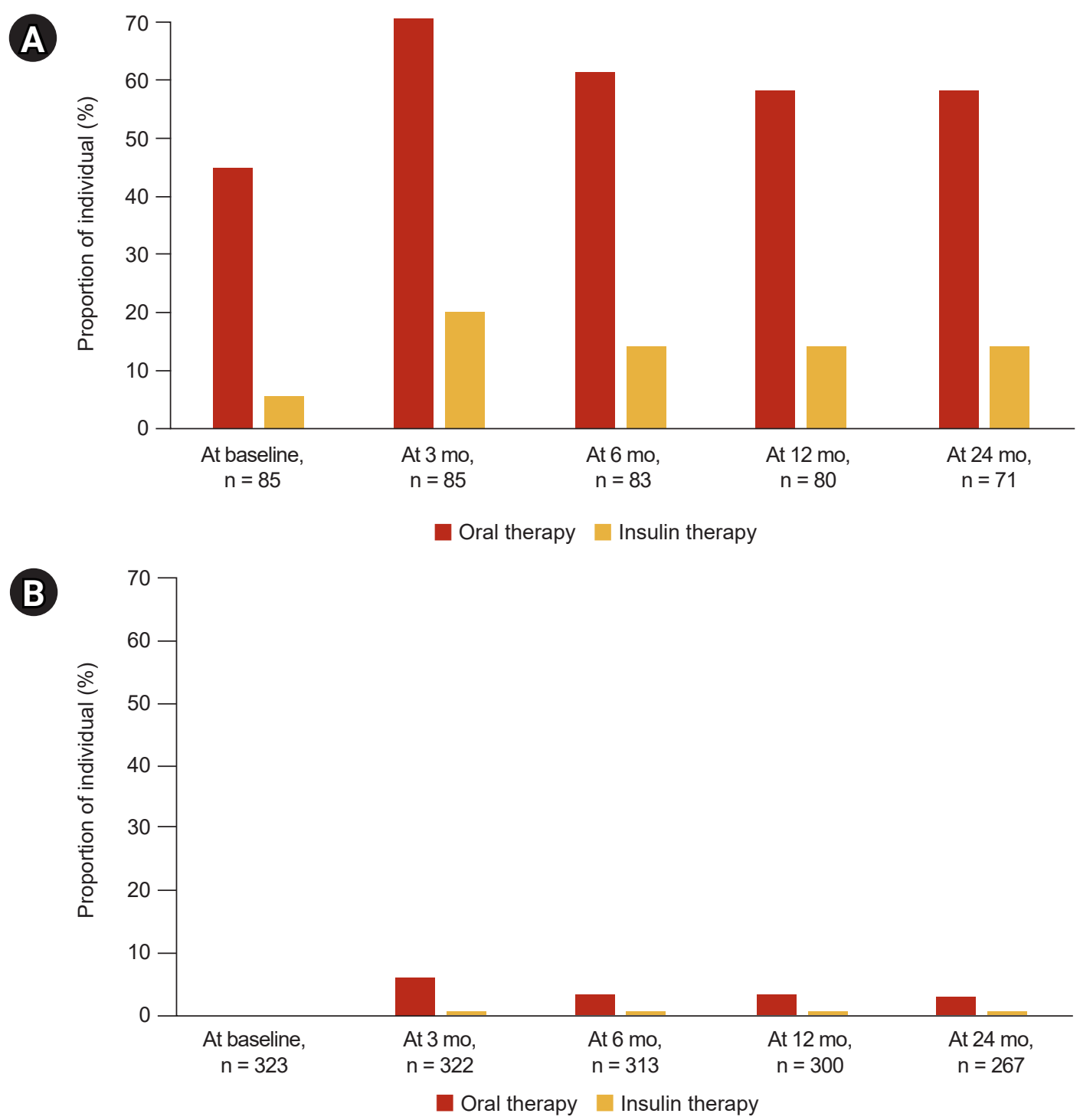

Figure 1. Proportion of individuals with or without diabetes mellitus (DM) at baseline who required antidiabetic medications during immunosuppressive therapy. (A) With DM. (B) Without DM.

risk of cardiovascular-related hospitalization than lupus nephritis, the inverse was true for mortality risk. Baseline ischemic heart disease increased the risk of cardiovascular-related hospitalization. A higher eGFR reduced the risks of all three adverse clinical events. While greater proteinuria increased the risk of ESKD, treatment with immunosuppressant reduced it. None of the covariates were significantly correlated with any other.

\section{Subgroup analyses by diabetes mellitus status at baseline}

In the DM group, older age (adjusted HR, 0.91; 95\% CI,
0.86-0.96; $\mathrm{p}=0.001$ ), higher eGFR (adjusted HR, 0.92; 95\% CI, 0.88-0.96; $\mathrm{p}<0.001$ for per $1 \mathrm{~mL} / \mathrm{min} / 1.73 \mathrm{~m}^{2}$ ), and treatment with ACE inhibitor or ARB (adjusted HR, 0.80; 95\% CI, 0.02-0.32; $\mathrm{p}<0.001$ ) were independently associated with reduced risk of ESKD. Higher eGFR (adjusted HR, 0.98; 95\% CI, 0.96-0.99; $\mathrm{p}=0.02$ for per $\mathrm{mL} / \mathrm{min} / 1.73 \mathrm{~m}^{2}$ ) and ACE inhibitor or ARB therapy (adjusted HR, 0.22; 95\% CI, 0.05-0.86; $p=0.03$ ) were associated with reduced risk of cardiovascular-related hospitalization, while baseline ischemic heart disease was associated with increased risk (adjusted HR, 6.94; 95\% CI, 2.64-18.25; $\mathrm{p}<0.001$ ). Concurrent histological diabetic nephropathy was not a significant predictor 


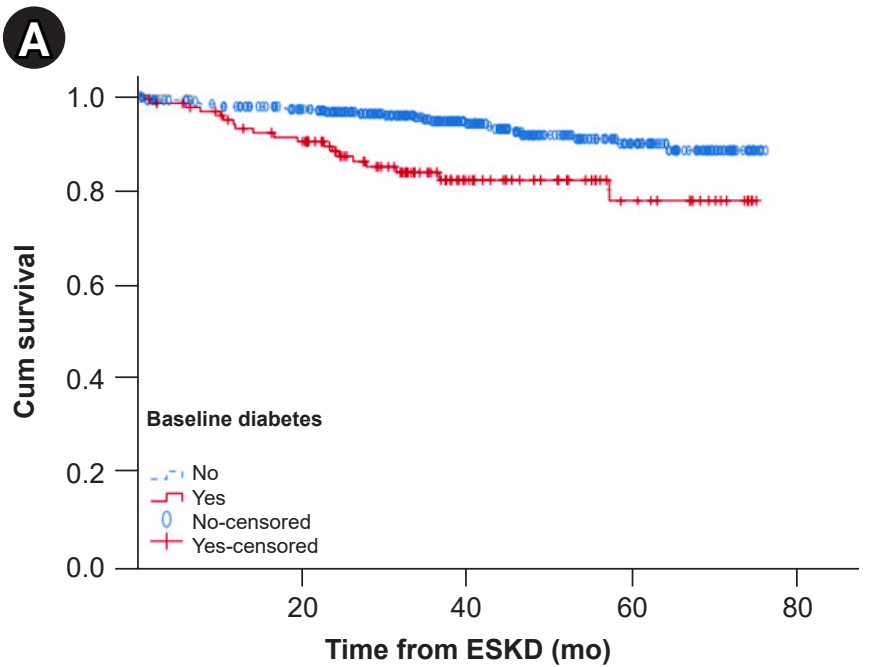

B
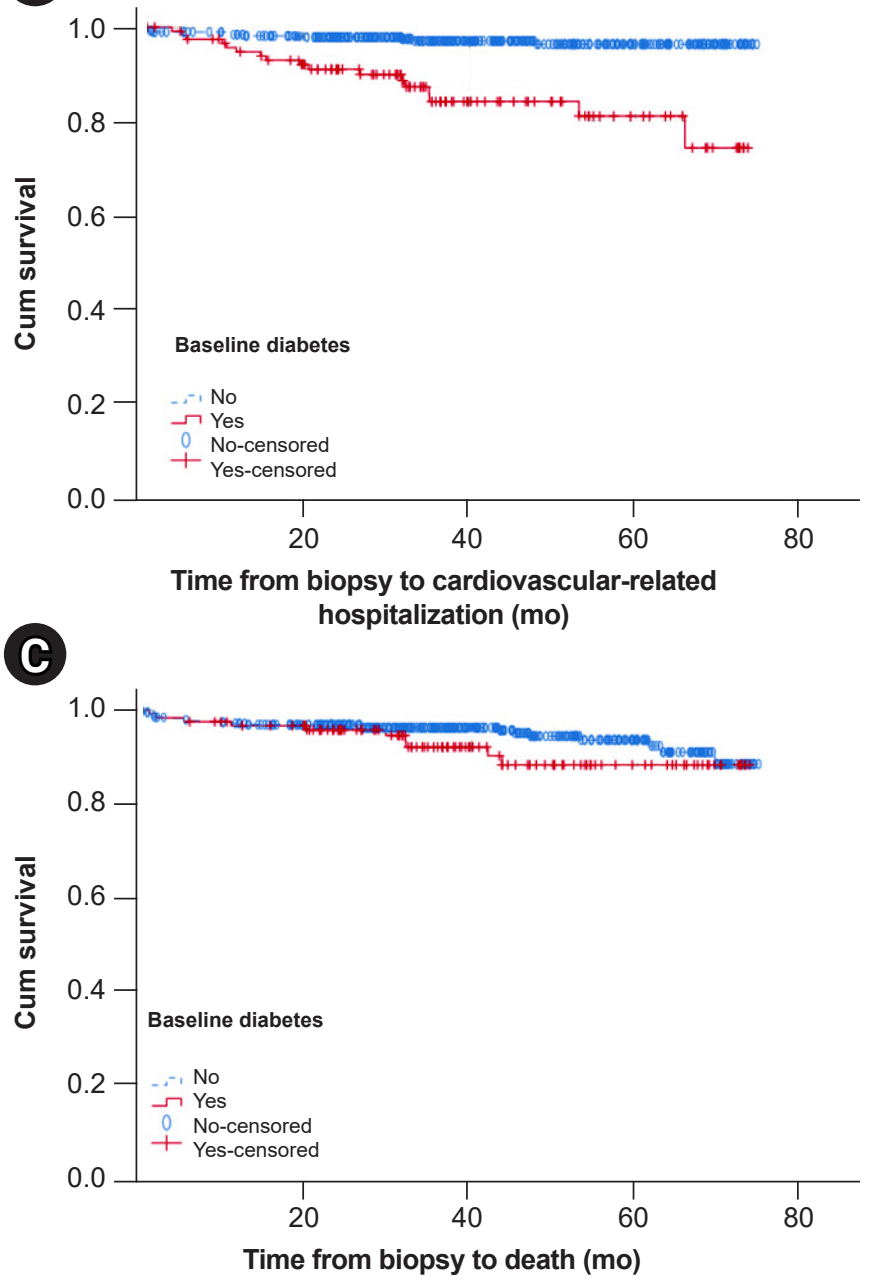

Figure 2. Kaplan-Meier survival curves. They show that cumulative probabilities of survival without the event were significantly different for (A) end-stage kidney disease (ESKD; log rank $p$ $=0.001$ ) and $(\mathrm{B})$ cardiovascular-related hospitalization (log rank $\mathrm{p}$ $<0.001)$, but not for $(C)$ mortality (log rank $p=0.22$ ). of ESKD or cardiovascular hospitalization when added as a variable in the multivariable models. Multivariate analysis was not performed for mortality in DM due to the small number of events.

In the no-DM group, eGFR was the only independent factor associated with ESKD (adjusted HR, 0.95; 95\% CI, 0.93-0.97; $\mathrm{p}<0.001$ ). Multivariate analysis was not performed for cardiovascular-related hospitalization due to the small number of events. Older age (adjusted HR, 1.09; 95\% CI, 1.05-1.14; $\mathrm{p}<0.001$ ), male sex (adjusted HR, 3.46; 95\% CI, 1.39-8.66; $\mathrm{p}=0.008$ ), and lupus nephritis (adjusted HR, 18.64; 95\% CI, 2.37-146.54; $\mathrm{p}=0.005$ ) were independently associated with increased mortality risk.

\section{Discussion}

DM was frequent among our multiethnic cohort of 601 individuals with biopsy-proven glomerulonephritis and renal vasculitis and was associated with worsening of the metabolic profile after immunosuppressant treatment. After accounting for age, sex, glomerulonephritis diagnosis, hypertension, hyperlipidemia, baseline ischemic heart disease, eGFR, proteinuria, and treatment with ACE inhibitor or ARB and immunosuppressants, DM was also found to be associated with a one-fold greater risk of ESKD and 1.7-fold greater risk of cardiovascular-related hospitalization in glomerulonephritis and renal vasculitis.

While DM is an established risk factor for kidney failure and cardiovascular disease in the general population and in individuals with chronic kidney disease [13,22-24], there has been little focus on the additive risks of DM in glomerulonephritis and renal vasculitis, which may in themselves result in inherently increased risks of ESKD and cardiovascular disease $[25,26]$. We confirmed that DM doubled the risk of cardiovascular-related hospitalization independent of other cardiovascular risk factors and underlying glomerulonephritis diagnosis. The few prior studies that evaluated the cardiovascular risks of DM in glomerulonephritis generally limited their cohorts to individuals with a single glomerulonephritis diagnosis and reported conflicting results. DM was associated with increased risk of cardiovascular events (age-adjusted HR, 7.07; 95\% CI, 1.88-26.54) in a medical records review of 58 incident ANCA-associated vasculitis [27], while a larger cohort of 504 Chinese patients with ANCA-associated vasculitis did not find DM to be associated 
Table 3. Factors associated with ESKD during treatment and follow-up of 511 individuals with lupus nephritis, ANCA-associated vasculitis, MCD or FSGS, membranous nephropathy, and IgA nephropathy

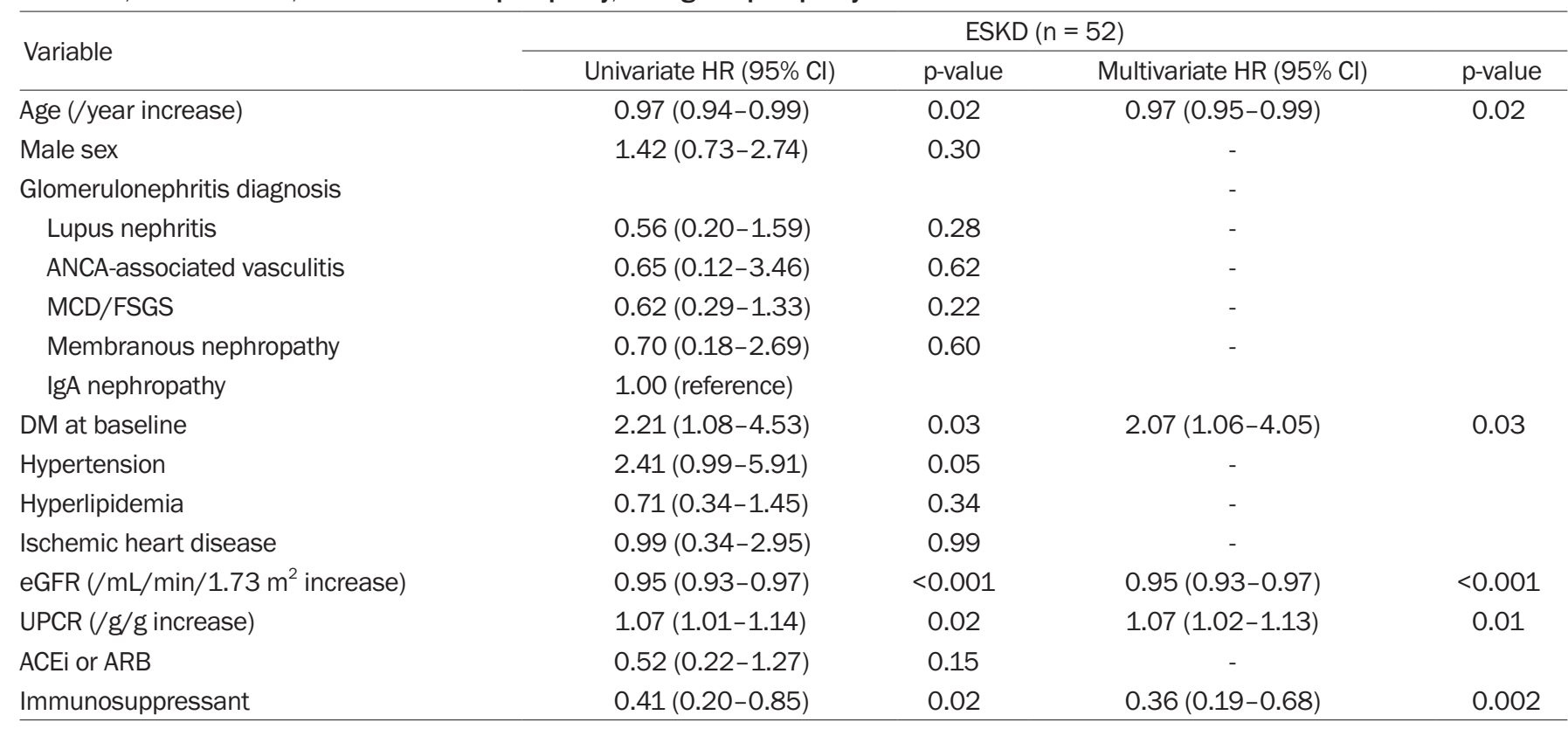

ACEi, angiotensin converting enzyme inhibitor; ANCA, anti-neutrophil cytoplasmic antibody; ARB, angiotensin II receptor blocker; Cl, confidence interval; DM, diabetes mellitus; eGFR, estimated glomerular filtration rate; ESKD, end-stage kidney disease; FSGS, focal segmental glomerulosclerosis; HR, hazard ratio; IgA, immunoglobulin A; MCD, minimal change disease; UPCR, urine protein-to-creatinine ratio.

with cardiovascular events [16]. Notably, a meta-analysis of observational studies on cardiovascular events in ANCA-associated vasculitis stressed that most early studies published before 2016 did not provide adequate information on traditional cardiovascular risk factors [28]. In a prospective cohort of 299 systemic lupus erythematosus (SLE) patients in North America [29], DM was associated with increased odds of coronary artery disease (univariate odds ratio [OR], 4.63; 95\% CI, 2.75-7.82; $\mathrm{p}=0.02$ ). In contrast, DM did not predict cardiovascular events among 277 SLE patients in Sweden who were followed up over 7 years [30]. A systematic review that evaluated 28 studies from the PubMed database from inception to 2012 highlighted that many cohort studies did not examine DM as a risk factor, while the few that did had small numbers of individuals with DM and did not find DM to be an independent risk factor for cardiovascular disease [31]. Among 221 patients with IgA nephropathy, DM was correlated with coronary heart disease in univariate analysis (OR, 5.87; 95\% CI, 1.61-21.5; p < 0.05), but not after adjusting for other vascular risk factors.

DM causes endothelial dysfunction and inflammation and thus vascular disease [32]. We also considered the plausibility that pre-existing DM exacerbated the metabolic derangements associated with immunosuppressant therapy in glomerulonephritis, thereby increasing cardiovascular risk. In particular, glucocorticosteroid was administered to $95.8 \%$ of patients treated with immunosuppressive therapy as monotherapy or in combination with other immunosuppressant and is associated with adverse changes in blood pressure, body weight, insulin resistance, and DM [10,12,33], which are traditional risk factors for cardiovascular disease $[13,14,33]$. While baseline DM was associated with significantly worse lipid indices after treatment with immunosuppressants, we did not identify immunosuppressant therapy as an independent risk factor for adverse cardiovascular events. However, prior studies of individuals with inflammatory and autoimmune diseases treated with moderate to high-dose glucocorticosteroid found increased risk of myocardial infarction and symptomatic coronary artery disease $[10,34,35]$. The association between low-dose glucocorticosteroid and cardiovascular events was less clear [36,37]. Interestingly, steroid-sparing or steroid-minimization regimens have been gaining traction in recent years [38,39]. In a randomized controlled trial of 176 individuals with IgA nephropathy, mycophenolate mofetil combined with reduced prednisolone dose was associated with lower risk of new-onset DM than 


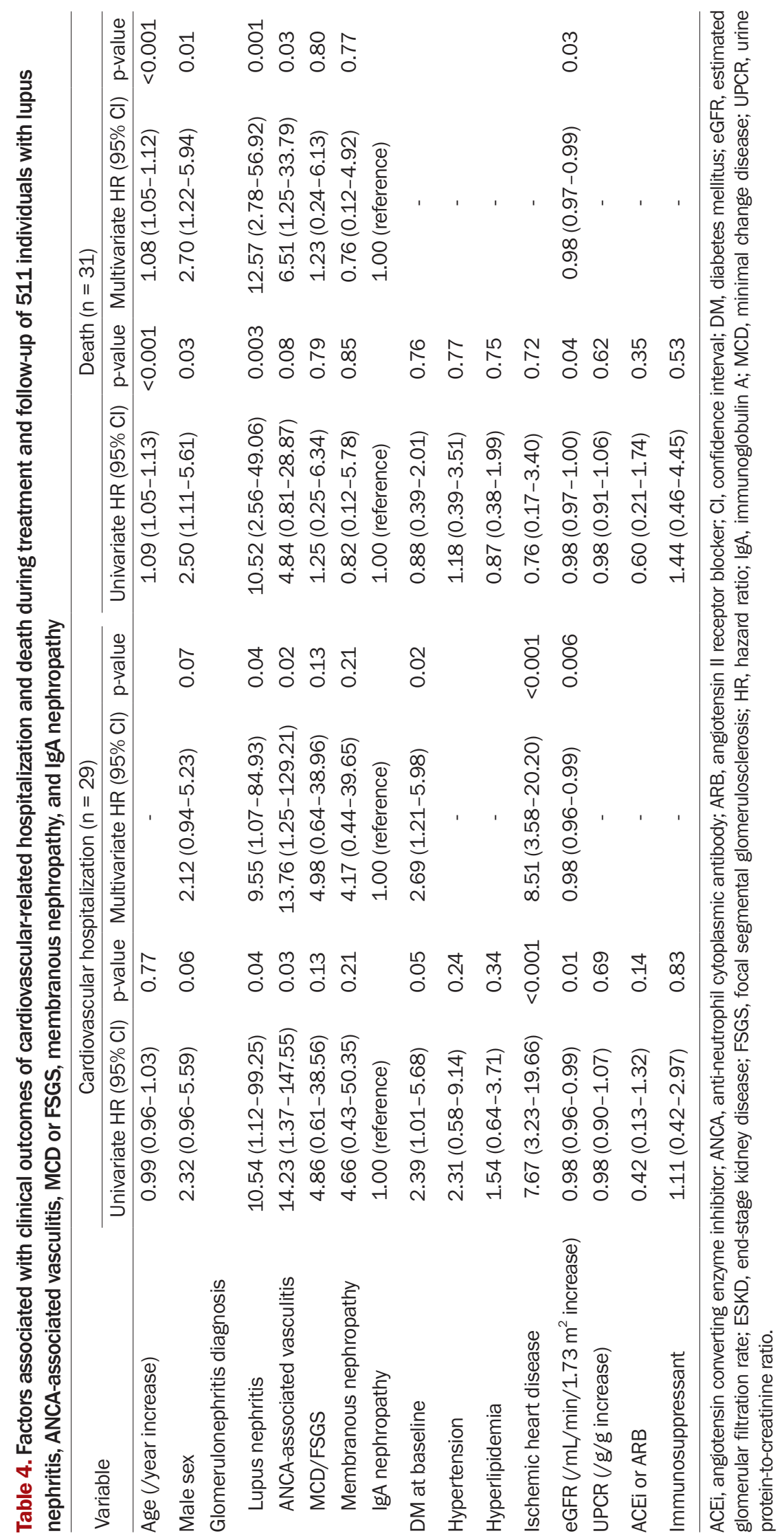


full-dose prednisolone ( $1 \%$ vs. $14 \%$ respectively, $\mathrm{p}=0.002$ ). Rapid prednisolone taper in the Plasma Exchange and Glucocorticoids in Severe ANCA-Associated Vasculitis (PEXIVAS) trial was comparable in efficacy to standard, higher-dose prednisolone but did not reduce the risk of endocrine adverse events [40]. While there are no long-term data on the cardiovascular effects of steroid-minimization therapies in glomerulonephritis, steroid avoidance and withdrawal therapies in renal transplantation were associated with significant cardiovascular risk reduction [41].

In this study, we also found that cardiovascular risk was increased inherently by ANCA-associated vasculitis and lupus nephritis, consistent with previous evidence $[15,25,26,28,31,42]$. The Joint European League Against Rheumatism and European Renal Association-European Dialysis and Transplant Association (EULAR/ERA-EDTA) noted in 2017 that while hypertension and DM were prevalent among patients with ANCA-associated vasculitis, the cardiovascular risk appeared to be greater than that conferred by traditional risk factors alone [26]. A subsequent meta-analysis of observational studies that compared ANCA-associated vasculitis with the general population or controls with chronic kidney disease found that ANCA-associated vasculitis had relative risks of 1.65 (95\% CI, 1.23-2.22) for all cardiovascular events and 1.60 (95\% CI, 1.39-1.84) for ischemic heart disease [28]. In SLE, the increased risk of cardiovascular disease has been attributed to accelerated atherosclerosis and presence of antiphospholipid antibodies [31,43]. A nested case-control study of 52,676 patients with SLE and 758,034 matched patients without SLE found that SLE was associated with greater odds of atherosclerotic cardiovascular disease (adjusted OR, 1.46; 95\% CI, 1.41-1.51) [42]. The aforementioned systematic review summarized epidemiologic data that SLE had at least 2- to 3-fold elevated risks of myocardial infarction, congestive heart failure, and overall cardiovascular mortality compared to the general population [31].

While DM increased the risk of ESKD in this study, evidence from the available literature is conflicting. An early retrospective cohort of 536 French patients with FSGS, membranous nephropathy, and IgA nephropathy followed up for a mean of 7 years found that DM was strongly associated with ESKD (adjusted HR, 2.6; 95\% CI, 1.2-5.8) [44]. However, a subsequent retrospective cohort of 580 Taiwanese patients diagnosed with membranous nephropathy, MCD, FSGS, and IgA nephropathy and followed up over a median of 5.9 years did not find DM to be a risk factor for ESKD [45]. Unfortunately, two large retrospective cohorts of primary glomerulonephritis, one of 1,943 membranous nephropathy, MCD, FSGS, IgA nephropathy, and membranoproliferative glomerulonephritis patients in Korea and another of 2,350 with membranous nephropathy, MCD, FSGS, IgA nephropathy, and lupus nephritis patients in South California, did not analyze DM as a risk factor for ESKD $[46,47]$. A Taiwanese National Health Insurance Research Database study compared 1,317 SLE patients with DM and propensity score-matched controls without DM who were followed up for a mean of 5 years and found that ESKD was higher in the DM group (incidence rate ratio, $2.71 ; 95 \% \mathrm{CI}, 1.70-4.32$ ) [48].

Interestingly, treatment with ACE inhibitor or ARB was associated with reduced risk of cardiovascular-related hospitalizations and ESKD among our patients with glomerulonephritis and renal vasculitis with DM. Previous systematic reviews of cardiovascular risk in ANCA vasculitis and SLE did not evaluate these medications as risk factors [28,31]. Similarly, these medications were not analyzed as risk factors for ESKD in a Taiwanese cohort of primary glomerulonephritis patients [45], while baseline ACE inhibitor or ARB was not associated with ESKD in a French cohort of primary glomerulonephritis patients (adjusted HR, 0.9; 95\% CI, 0.5-1.6) [44]. However, both ACE inhibitor and ARB reduced major cardiovascular events (OR, 0.82; 95\% credible interval, 0.71-0.92 and OR 0.76; 95\% credible interval, 0.62-0.89, respectively) and kidney failure (OR, 0.61; 95\% credible interval, 0.47-0.79 and OR, 0.70; 95\% credible interval, 0.52-0.89, respectively) compared to placebo in a meta-analysis of 119 randomized controlled trials of 64,768 participants with chronic kidney disease $[49,50]$.

Our study had several limitations. We did not retroactively adjudicate the diagnosis of primary FSGS. However, the glomerulonephritis diagnosis was corroborated with the physician-documented clinical diagnosis, which took into consideration clinical and histologic features that supported the diagnosis [51], including the degree of podocyte foot effacement routinely reported by our pathologists, and that were used to differentiate primary from secondary FSGS [52]. The impacts of ACE inhibitor, ARB, and immunosuppressants on clinical outcomes may be confounded by indications, while the effect of DM may be confounded by other cardiovascular risk modifiers such as smoking, use of antiplatelet or anticoagulation drugs, and statins, which were not included in this 
observational study. Thus, causality cannot be confirmed, and the results of this study are only intended for hypothesis-generation. The small number of cardiovascular events may limit the power to detect significant associations and likely contributed to imprecise risk estimates with large CIs. Similarly, the absence of significant interactions may also be due to the lack of power to detect heterogeneity in the effects measured [53]. While we found qualitative heterogeneity in the risk effect as nephrotic-range proteinuria was an effect modifier for the time to ESKD analysis [53], we acknowledge that multiple analyses may be prone to Type 1 errors. Lastly, the results of our study, obtained in a relatively old population with high prevalence of traditional cardiovascular risk factors, may not be generalizable to other cohorts with different demographic and clinical profiles.

Despite its limitations, this study has several strengths such as a long observation period sufficient for development of the outcomes of interest and the use of multivariate regression analysis to adjust for imbalances in risk factors between exposure groups. Importantly, we identified risk factors for long-term renal and cardiovascular outcomes among individuals with biopsy-proven glomerulonephritis and renal vasculitis, including modifiable factors amenable to interventions to reduce adverse events. Considering our findings and the current evidence, we propose that individuals with DM and biopsy-proven glomerulonephritis should be carefully and regularly assessed for cardiovascular risk and have their modifiable risk factors optimized. Patients with DM or at high cardiovascular risk may benefit from ACE inhibitor or ARB therapy and consideration for steroid-minimization immunosuppressive regimens, but further research will be required to evaluate the long-term cardiovascular benefits of such strategies.

\section{Conflicts of interest}

Jason C. J. Choo has served on Advisory Boards for Novartis and Pfizer and has donated honoraria to Singapore General Hospital to support research and education.

\section{Acknowledgments}

The authors would like to thank their colleagues in the Department of Renal Medicine for their support of this project.

\section{Authors' contributions}

Conceptualization, Data curation, Methodology, Project administration: CCL, JCJC

Formal analysis: CCL

Investigation: THZ, IM, CYM, CCM, WKT

Writing-original draft: CCL

Writing-review \& editing: All authors

All authors read and approved the final manuscript.

\section{ORCID}

Cynthia C. Lim, https://orcid.org/0000-0003-0021-4861

Jason C. J. Choo, https://orcid.org/0000-0002-8477-0875

Hui Zhuan Tan, https://orcid.org/0000-0003-4899-6399

Irene Y. J. Mok, https://orcid.org/0000-0001-6796-6414

Yok Mooi Chin, https://orcid.org/0000-0002-2156-5137 Choong Meng Chan, https://orcid.org/0000-0002-3297-0218

Keng Thye Woo, https://orcid.org/0000-0001-7051-1728

\section{References}

1. Haynes R, Staplin N, Emberson J, et al. Evaluating the contribution of the cause of kidney disease to prognosis in CKD: results from the Study of Heart and Renal Protection (SHARP). Am J Kidney Dis 2014;64:40-48.

2. Wakai K, Nakai S, Kikuchi K, et al. Trends in incidence of endstage renal disease in Japan, 1983-2000: age-adjusted and age-specific rates by gender and cause. Nephrol Dial Transplant 2004;19:2044-2052.

3. Yang WC, Hwang SJ; Taiwan Society of Nephrology. Incidence, prevalence and mortality trends of dialysis end-stage renal disease in Taiwan from 1990 to 2001: the impact of national health insurance. Nephrol Dial Transplant 2008;23:3977-3982.

4. GBD 2017 Causes of Death Collaborators. Global, regional, and national age-sex-specific mortality for 282 causes of death in 195 countries and territories, 1980-2017: a systematic analysis for the Global Burden of Disease Study 2017. Lancet 2018;392:17361788.

5. Cunningham A, Benediktsson $\mathrm{H}$, Muruve DA, Hildebrand AM, Ravani P. Trends in biopsy-based diagnosis of kidney disease: a population study. Can J Kidney Health Dis 2018;5: 2054358118799690.

6. Zink CM, Ernst S, Riehl J, et al. Trends of renal diseases in Germany: review of a regional renal biopsy database from 1990 to 
2013. Clin Kidney J 2019;12:795-800.

7. Woo KT, Chan CM, Lim C, et al. A global evolutionary trend of the frequency of primary glomerulonephritis over the past four decades. Kidney Dis (Basel) 2019;5:247-258.

8. Zhou FD, Zhao MH, Zou WZ, Liu G, Wang H. The changing spectrum of primary glomerular diseases within 15 years: a survey of 3331 patients in a single Chinese centre. Nephrol Dial Transplant 2009;24:870-876.

9. Kim K, Lee SH, Lee SW, Lee JP, Chin HJ; Korean GlomeruloNEphritis sTudy (KoGNET) Group. Current findings of kidney biopsy including nephropathy associated with hypertension and diabetes mellitus in Korea. Korean J Intern Med 2020;35:1173-1187.

10. Rice JB, White AG, Scarpati LM, Wan G, Nelson WW. Long-term systemic corticosteroid exposure: a systematic literature review. Clin Ther 2017;39:2216-2229.

11. Sarnes E, Crofford L, Watson M, Dennis G, Kan H, Bass D. Incidence and US costs of corticosteroid-associated adverse events: a systematic literature review. Clin Ther 2011;33:1413-1432.

12. Lim CC, Gardner D, Ng RZ, et al. Synergistic impact of pre-diabetes and immunosuppressants on the risk of diabetes mellitus during treatment of glomerulonephritis and renal vasculitis. Kidney Res Clin Pract 2020;39:172-179.

13. Lim CC, Teo BW, Ong PG, et al. Chronic kidney disease, cardiovascular disease and mortality: a prospective cohort study in a multi-ethnic Asian population. Eur J Prev Cardiol 2015;22:10181026.

14. Global Burden of Metabolic Risk Factors for Chronic Diseases Collaboration. Cardiovascular disease, chronic kidney disease, and diabetes mortality burden of cardiometabolic risk factors from 1980 to 2010: a comparative risk assessment. Lancet Diabetes Endocrinol 2014;2:634-647.

15. Morgan MD, Turnbull J, Selamet U, et al. Increased incidence of cardiovascular events in patients with antineutrophil cytoplasmic antibody-associated vasculitides: a matched-pair cohort study. Arthritis Rheum 2009;60:3493-3500.

16. Bai YH, Li ZY, Chang DY, Chen M, Kallenberg CG, Zhao MH. The BVAS is an independent predictor of cardiovascular events and cardiovascular disease-related mortality in patients with ANCA-associated vasculitis: a study of 504 cases in a single Chinese center. Semin Arthritis Rheum 2018;47:524-529.

17. Flossmann O, Berden A, de Groot K, et al. Long-term patient survival in ANCA-associated vasculitis. Ann Rheum Dis 2011; 70:488-494.

18. Faurschou M, Dreyer L, Kamper AL, Starklint H, Jacobsen S. Long-term mortality and renal outcome in a cohort of 100 pa- tients with lupus nephritis. Arthritis Care Res (Hoboken) 2010; 62:873-880.

19. Yap DY, Tang CS, Ma MK, Lam MF, Chan TM. Survival analysis and causes of mortality in patients with lupus nephritis. Nephrol Dial Transplant 2012;27:3248-3254.

20. Knoop T, Vikse BE, Svarstad E, Leh S, Reisæter AV, Bjørneklett R. Mortality in patients with IgA nephropathy. Am J Kidney Dis 2013;62:883-890.

21. Levey AS, Stevens LA, Schmid CH, et al. A new equation to estimate glomerular filtration rate. Ann Intern Med 2009;150: 604-612.

22. Lim CC, Chee ML, Cheng CY, et al. Simplified end stage renal failure risk prediction model for the low-risk general population with chronic kidney disease. PLoS One 2019;14:e0212590.

23. Lee WL, Cheung AM, Cape D, Zinman B. Impact of diabetes on coronary artery disease in women and men: a meta-analysis of prospective studies. Diabetes Care 2000;23:962-968.

24. Woodward M, Zhang X, Barzi F, et al. The effects of diabetes on the risks of major cardiovascular diseases and death in the Asia-Pacific region. Diabetes Care 2003;26:360-366.

25. Fanouriakis A, Kostopoulou M, Alunno A, et al. 2019 update of the EULAR recommendations for the management of systemic lupus erythematosus. Ann Rheum Dis 2019;78:736-745.

26. Yates M, Watts RA, Bajema IM, et al. EULAR/ERA-EDTA recommendations for the management of ANCA-associated vasculitis. Ann Rheum Dis 2016;75:1583-1594.

27. Berti A, Matteson EL, Crowson CS, Specks U, Cornec D. Risk of cardiovascular disease and venous thromboembolism among patients with incident ANCA-associated vasculitis: a 20 -year population-based cohort study. Mayo Clin Proc 2018;93:597606.

28. Houben E, Penne EL, Voskuyl AE, et al. Cardiovascular events in anti-neutrophil cytoplasmic antibody-associated vasculitis: a meta-analysis of observational studies. Rheumatology (Oxford) 2018;57:555-562.

29. Petri M. Detection of coronary artery disease and the role of traditional risk factors in the Hopkins Lupus Cohort. Lupus 2000;9:170-175.

30. Bengtsson C, Ohman ML, Nived O, Rantapää Dahlqvist S. Cardiovascular event in systemic lupus erythematosus in northern Sweden: incidence and predictors in a 7-year follow-up study. Lupus 2012;21:452-459.

31. Schoenfeld SR, Kasturi S, Costenbader KH. The epidemiology of atherosclerotic cardiovascular disease among patients with SLE: a systematic review. Semin Arthritis Rheum 2013;43:77-95. 
32. Paneni F, Beckman JA, Creager MA, Cosentino F. Diabetes and vascular disease: pathophysiology, clinical consequences, and medical therapy. Part I. Eur Heart J 2013;34:2436-2443.

33. Fardet L, Fève B. Systemic glucocorticoid therapy: a review of its metabolic and cardiovascular adverse events. Drugs 2014;74:1731-1745.

34. Zonana-Nacach A, Barr SG, Magder LS, Petri M. Damage in systemic lupus erythematosus and its association with corticosteroids. Arthritis Rheum 2000;43:1801-1808.

35. Roubille C, Richer V, Starnino T, et al. The effects of tumour necrosis factor inhibitors, methotrexate, non-steroidal anti-inflammatory drugs and corticosteroids on cardiovascular events in rheumatoid arthritis, psoriasis and psoriatic arthritis: a systematic review and meta-analysis. Ann Rheum Dis 2015; 74:480-489.

36. Ruyssen-Witrand A, Fautrel B, Saraux A, Le Loët X, Pham T. Cardiovascular risk induced by low-dose corticosteroids in rheumatoid arthritis: a systematic literature review. Joint Bone Spine 2011;78:23-30.

37. Souverein PC, Berard A, Van Staa TP, et al. Use of oral glucocorticoids and risk of cardiovascular and cerebrovascular disease in a population based case-control study. Heart 2004;90:859-865.

38. Walsh M, Merkel PA, Peh CA, et al. Plasma exchange and glucocorticoid dosing in the treatment of anti-neutrophil cytoplasm antibody associated vasculitis (PEXIVAS): protocol for a randomized controlled trial. Trials 2013;14:73.

39. Hou JH, Le WB, Chen N, et al. Mycophenolate mofetil combined with prednisone versus full-dose prednisone in IgA nephropathy with active proliferative lesions: a randomized controlled trial. Am J Kidney Dis 2017;69:788-795.

40. Walsh M, Merkel PA, Peh CA, et al. Plasma exchange and glucocorticoids in severe ANCA-associated vasculitis. $N$ Engl J Med 2020;382:622-631.

41. Knight SR, Morris PJ. Steroid avoidance or withdrawal after renal transplantation increases the risk of acute rejection but decreases cardiovascular risk. A meta-analysis. Transplantation 2010;89:1-14.

42. Katz G, Smilowitz NR, Blazer A, Clancy R, Buyon JP, Berger JS.
Systemic lupus erythematosus and increased prevalence of atherosclerotic cardiovascular disease in hospitalized patients. Mayo Clin Proc 2019;94:1436-1443.

43. Haque S, Skeoch S, Rakieh C, et al. Progression of subclinical and clinical cardiovascular disease in a UK SLE cohort: the role of classic and SLE-related factors. Lupus Sci Med 2018;5:e000267.

44. Moranne O, Watier L, Rossert J, Stengel B; GN-Progress Study Group. Primary glomerulonephritis: an update on renal survival and determinants of progression. QJM 2008;101:215-224.

45. Chou YH, Lien YC, Hu FC, et al. Clinical outcomes and predictors for ESRD and mortality in primary GN. Clin J Am Soc Nephrol 2012;7:1401-1408.

46. Lee H, Kim DK, Oh KH, et al. Mortality and renal outcome of primary glomerulonephritis in Korea: observation in 1,943 biopsied cases. Am J Nephrol 2013;37:74-83.

47. Sim JJ, Bhandari SK, Batech M, et al. End-stage renal disease and mortality outcomes across different glomerulonephropathies in a large diverse US population. Mayo Clin Proc 2018;93:167-178.

48. Jiang MY, Hwang JC, Feng IJ. Impact of diabetes mellitus on the risk of end-stage renal disease in patients with systemic lupus erythematosus. Sci Rep 2018;8:6008.

49. Gansevoort RT, de Zeeuw D, de Jong PE. Long-term benefits of the antiproteinuric effect of angiotensin-converting enzyme inhibition in nondiabetic renal disease. Am J Kidney Dis 1993; 22:202-206.

50. Xie X, Liu Y, Perkovic V, et al. Renin-angiotensin system inhibitors and kidney and cardiovascular outcomes in patients with CKD: a Bayesian network meta-analysis of randomized clinical trials. Am J Kidney Dis 2016;67:728-741.

51. Sethi S, Glassock RJ, Fervenza FC. Focal segmental glomerulosclerosis: towards a better understanding for the practicing nephrologist. Nephrol Dial Transplant2015;30:375-384.

52. Sethi S, Zand L, Nasr SH, Glassock RJ, Fervenza FC. Focal and segmental glomerulosclerosis: clinical and kidney biopsy correlations. Clin Kidney J 2014;7:531-537.

53. Wang R, Lagakos SW, Ware JH, Hunter DJ, Drazen JM. Statistics in medicine: reporting of subgroup analyses in clinical trials. $N$ Engl J Med 2007;357:2189-2194. 